\title{
Computer-Assisted Reconstruction of the Hemimaxillectomy Defects With a Composite Iliac Crest Bone Free Flap: Case Reports
}

\author{
Sergey V. Tereshchuk, MD ${ }^{1 *}$; Sergey Y. Ivanov, MD²; Vladimir A. Sukharev, MD³ \\ ${ }^{1}$ Center for Maxillofacial Surgery and Dentistry, Burdenko Main Military Clinical Hospital, Moscow, Russia \\ I.M. Sechenov First Moscow State Medical University, Moscow, Russia \\ ${ }^{3}$ Department for Plastic and Reconstructive Surgery, Burdenko Main Military Clinical Hospital, Moscow, Russia
}

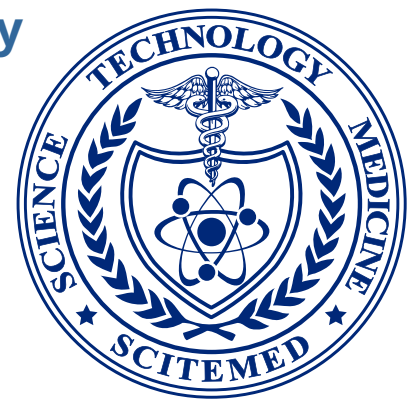

\begin{abstract}
Hemimaxillectomy defects incorporate the body of the maxilla and the orbital floor in the vertical dimension; the horizontal component includes half of the hard palate and alveolus without the involvement of the nasal septum. The goal of the treatment for the patients with maxillary defects is to restore a barrier between the oral cavity and the structures above it, re-establish the support to the soft tissue of the face, and restitute the dentition. In this article, we present two cases that illustrate the utilization of the iliac crest bone flap in combination with computer-assisted techniques for hemimaxillectomy defects reconstruction. It can give a support to an eye globe and be a basis for dental implants. The deep fascia and/ or the inner oblique muscle can be included into the flap to serve as an additional material for intraoral defects. The iliac crest bone flap cannot restore the contour of the maxilla and the neighboring cheekbone precisely. This disadvantage, as well as the position of the eye globe, can be easily corrected by means of customized implants.
\end{abstract}

\section{Introduction}

Reconstruction of the maxillary defects remains a matter of controversy. The maxilla has a complex three-dimensional shape. It bears teeth and is hollow inside. It separates nasal and oral cavities and gives support to an eye. The bone is covered with a relatively thin layer of fixed soft tissue. It can be affected by squamous cell carcinoma arising from the lining of the alveolar ridge, palate, nasal, and sinus cavity. It can also be affected by the malignant tumors of minor salivary glands densely spread over the palate, odontogenic and non-odontogenic neoplasms, as well as benign tumors. The modalities of the surgical treatment vary from a simple resection to the vast ablative surgeries limited only by dura mater [1].

The goal of the treatment for the patients with maxillary defects is to restore a barrier between the oral cavity and the structures above it, re-establish the support to the soft tissue of the face and the eye globe, and restitute the dentition. Class 3a hemimaxillary defects according to the Brown classification incorporate the body of the maxilla and the orbital floor in the vertical dimension; the horizontal component includes half of the hard palate and alveolus without the involvement of the nasal septum [2]. Approaches to the closure of these kinds of defects are obturators, regional flaps, and free flaps. In this article, we illustrate the novel utilization of the iliac crest bone flap in combination with computer-assisted techniques for hemimaxillectomy defects reconstruction $[3,4]$.

\section{Case Reports}

\section{Case 1}

A 33-year-old presented to our Center with a swelling of the right cheek. Physical examination yielded a painless lesion in the area of the anterior wall of the right maxilla with edema and erythema of overlying skin. The skin was fixed to the tumor. CT scanning demonstrated that the mass totally obstructing the right maxillary sinus had extended to the orbital floor, the anterior wall of the maxillary sinus, cheekbone, and the skin superior to it (Figure 1). For pre-operative morphological verification of the tumor, core needle biopsy was carried out. The pathological diagnosis of moderately differentiated squamous cells carcinoma had been demonstrated. The final decision was a neck dissection followed by a composite resection of the right maxilla with the overlying skin, the orbital floor, and the neighboring part of the cheekbone (Figure 2). The primary free flap reconstruction was considered with preference given to the iliac bone free flap. A virtual surgical planning and stereolithographic modeling preceded the operation. The facial skeleton and iliac bones were scanned.

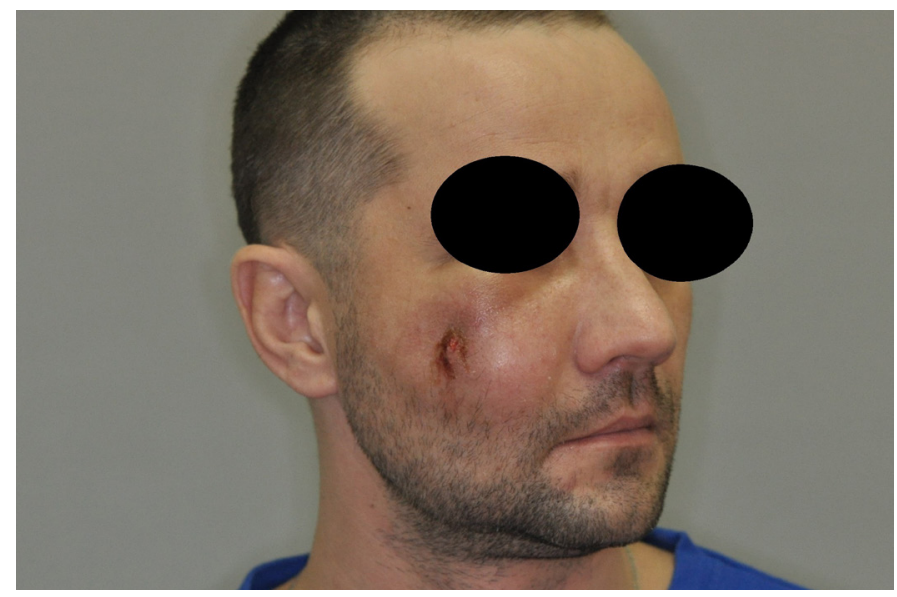

Figure 1. Appearance of the patient on admission.

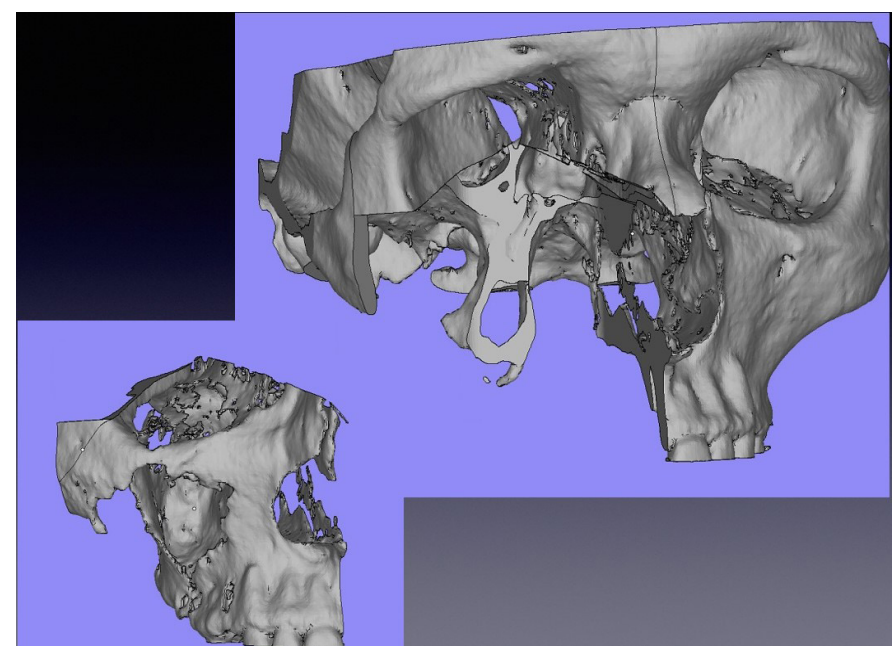

Figure 2. Planned hemimaxillectomy. 


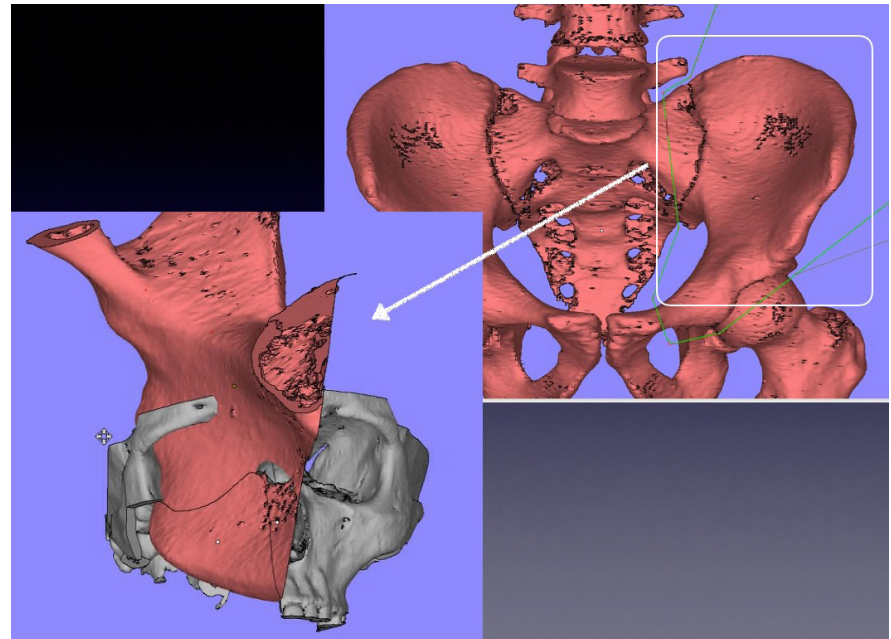

Figure 3. Donor site for the free flap.

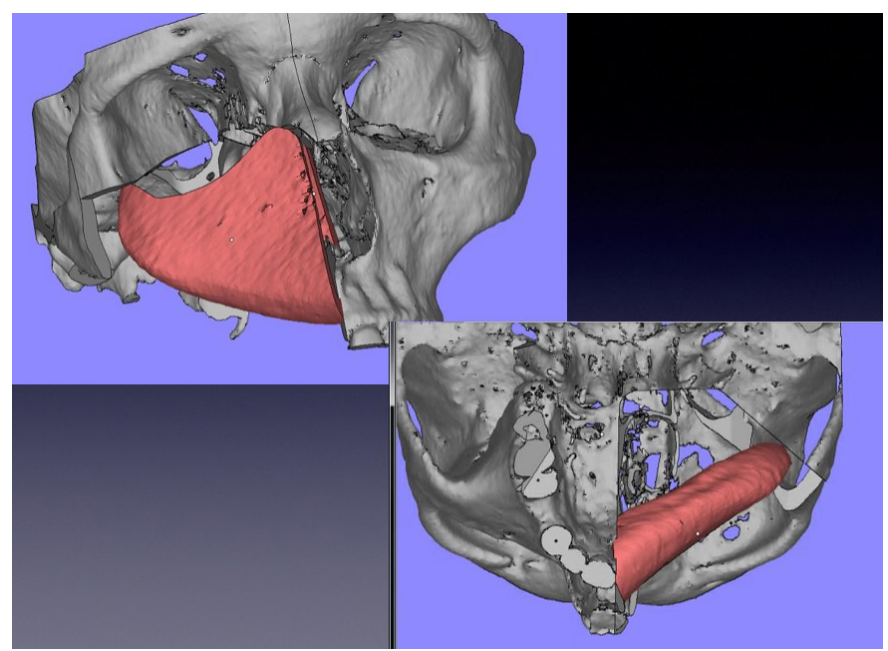

Figure 4. Designing and positioning of the iliac crest bone flap.

Three-dimensional model of the free iliac crest bone flap was placed into the maxillary defect in an upside-down position with its anterior superior spine directed laterally. Having taken into account the scar in the right groin region of the patient (Figure 3 ) and our desire to raise the iliac bone free flap with a portion of the inner oblique muscle, the left iliac seemed to be the more reliable donor site option than the right one. The peddle of the flap would run along its anterior surface behind the zygomatic arch toward the right superficial temporal artery and vein. The iliac bone was adjusted to replicate the to-be-taken-away alveolus and to match the opposing dentition of the mandible in the best way (Figure 4). The upper ridge of the flap bone crossed the orbit anteroposteriorly and mediolaterally so as to give support to the eye globe at the level of its equator. This ridge was planned to replicate the curvature of the orbital floor. We planned to fix the flap to the skull with plates at three points: nose bone, lateral orbital wall, and left alveolus. Stereolithographic cutting guides for the resection of the maxilla and the iliac bone osteotomy were designed and manufactured.

The maxilla was reached through the modified Webber-Ferguson approach. Frozen biopsy proved clear surgical margins. A coronoidectomy was carried out to prevent trismus. The iliac crest bone flap was raised with the inner oblique muscle on the ascending artery and a skin island of the groin region on the perforator artery. It should be noted here that we had planned to close the skin defect with the radial free flap. During the raising of the flap, we identified vessels running from the deep circumflex iliac artery toward the skin. So, we decided to include

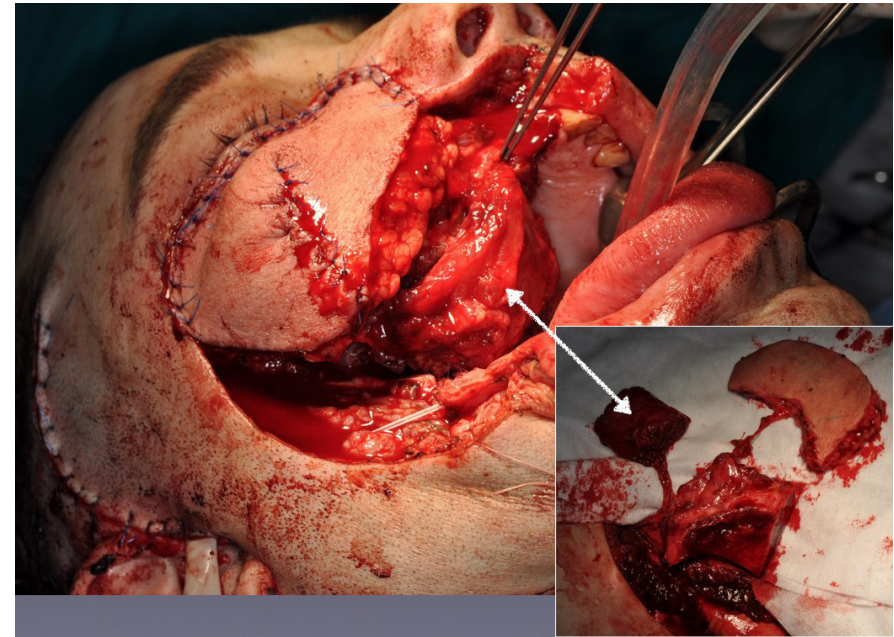

Figure 5. Closing of the intra oral defect with the portion of the inner oblique muscle of the flap.

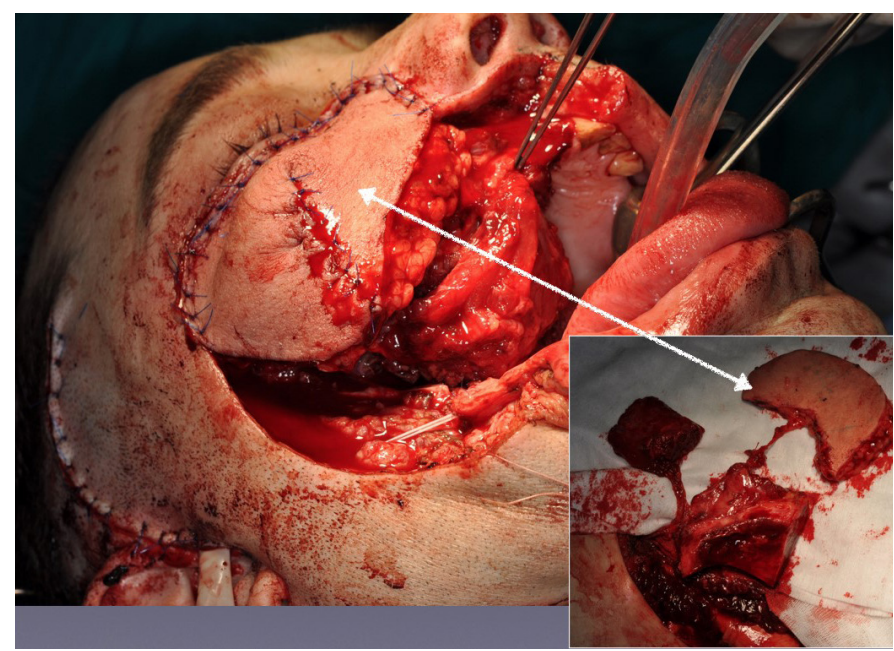

Figure 6. Closing of the skin defect with the skin island of the flap.

this skin island into the flap. Placement of the iliac bone into the defect left a gap at the palate that was closed with the portion of the inner oblique muscle (Figure 5). The defect of the skin was closed with the skin island of the flap (Figure 6).

One-month postoperative CT scanning of the skull of the patient was performed. The data were used to create a three-dimensional model that was compared with the model created during the virtual planning before the operation. The acquired data demonstrated good quality of the bone and its satisfactory positioning in the defect (Figure 7). The upper edge of the flap was at the same level as the orbital floor on the contralateral side, giving enough support to the right eye globe.

At this stage, it is not yet clear, but from our experience, it is quite obvious that the contour of the newly reconstructed maxilla won't match the left side. Contour refinement can be done by means of a customized contour implant. Our next patient can serve as a demonstration of the process.

\section{Case 2}

A 45-year-old male patient came to our Center with the defect of the left maxilla resulted from the hemimaxillectomy for sarcoma, eight months prior to the visit. The donor site choice, virtual planning of the operation, and its execution were similar to the previous case. Nine months after the operation, no recurrence of the tumor was observed. CT data showed good results of the operation (Figure 8). The bone flap healed 


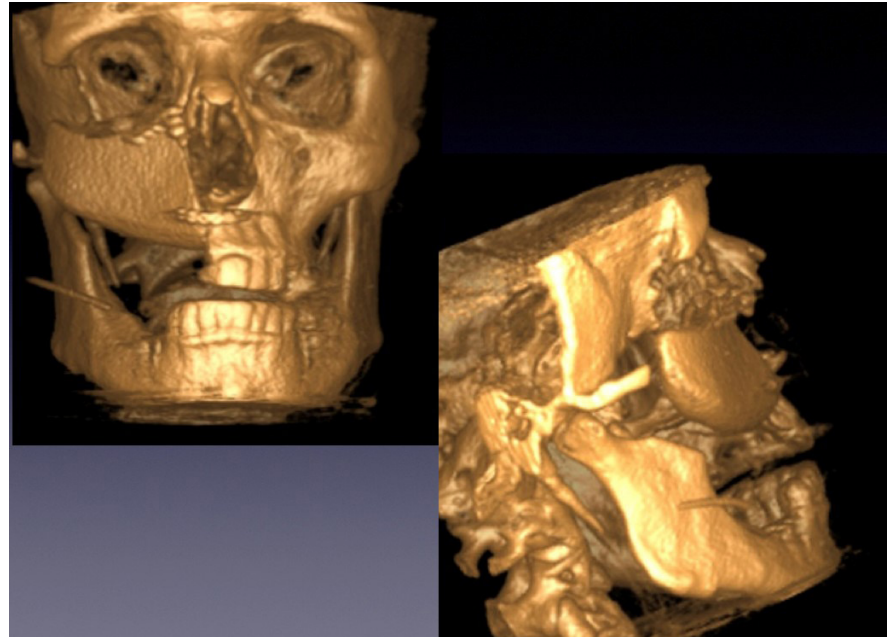

Figure 7. Three-dimensional reconstruction of the skull one month after the operation.

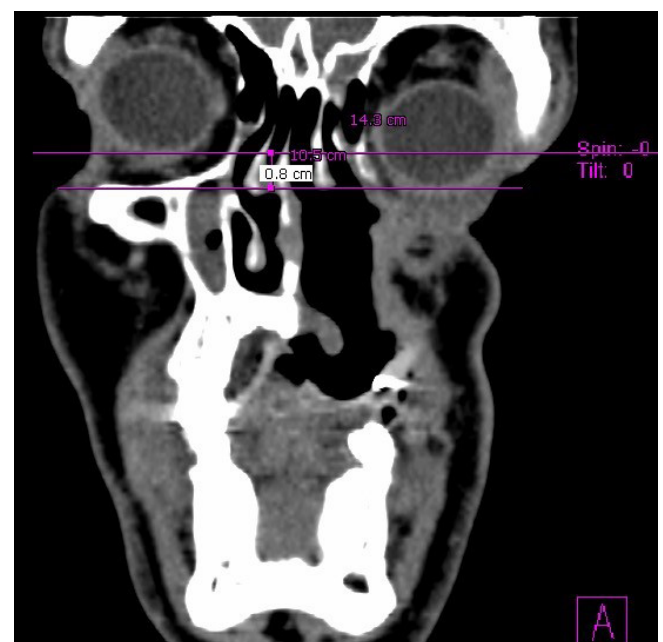

Figure 8. The left eye has sunk in the maxillary defect $8 \mathrm{~mm}$ lower than the right eye.

(Figure 9). The left eye globe, having got a support of the iliac bone flap, was in a relatively anatomical position. At the same time, the contour of the left cheek region wasn't satisfactory. To solve this problem, a virtual three-dimensional model of the skull was created. We applied a mirroring process to calculate the difference between two sides. Deduction of the virtual models resulted in a model of a contour implant (Figure 10). The implant then was prototyped of ultrahigh molecular weight polyethylene (Figure 11).

\section{Discussion}

The traditional approach to the closure of the maxillary defects after hemimaxillectomy is an obturator $[5,6]$. Disadvantages of the obturators include the need for manual dexterity in handling them, insufficient retention and adhesion resulting in fluid leakage into the nasal cavity, irritation of the adjacent soft tissue, and loss of the anchoring teeth. Over time, the patients become more discontented with their prostheses [7].

Any attempt to reconstruct the hemimaxillectomy defects with bone grafts or alloplastic bone substitutes is doomed due to an insufficient amount of soft tissue and poor wound-healing properties in the recipient site. Local and regional flaps for reconstruction of maxillary defects include the palatal island flap, the buccal fat pad, and the temporalis muscle flap. All these options are capable of restoring the oronasal barrier and smaller bony defects of the maxilla [8].

Advances in microvascular surgery over the past thirty years have

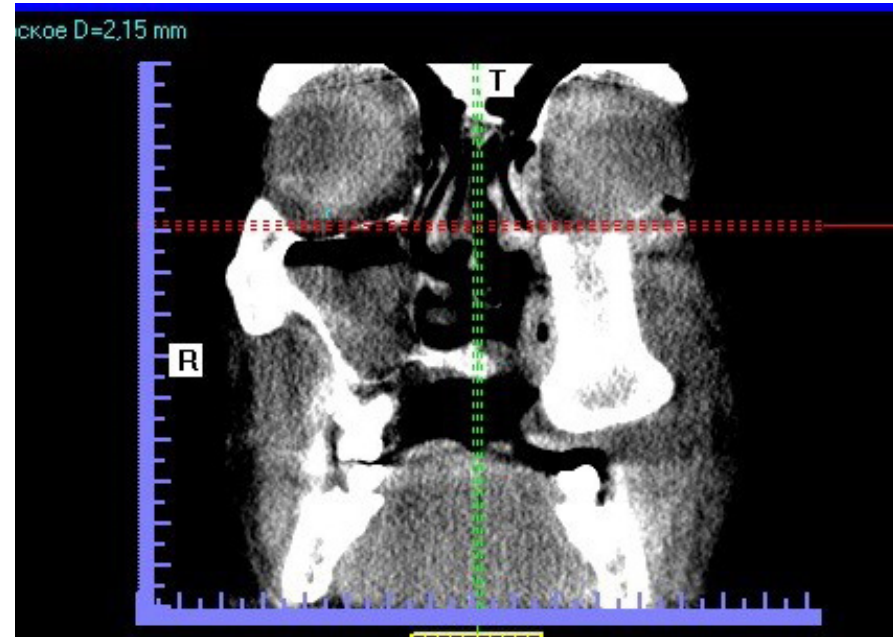

Figure 9. The iliac crest bone is supporting the left eye.

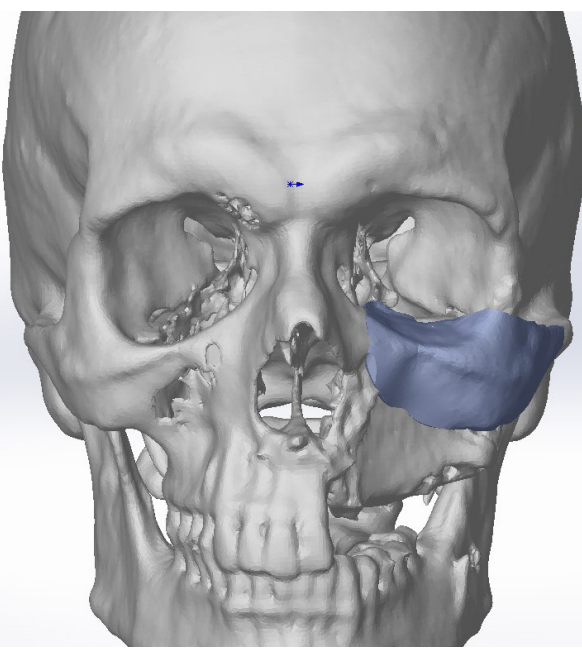

Figure 10. Three-dimensional model of the contour implant after the mirroring.

become state-of-the-art techniques for the face and neck reconstruction. Despite the vast armamentarium of the free flaps, the real options are scapula, fibula, rib, and iliac crest donor sites [9].

Subscapular artery system flaps or composite flaps with serratus anterior muscle and the angle of the scapula are versatile enough to reconstruct maxillary defects of various sizes. However, neither is suitable to provide support for a sinking eye or dental implants, and to separate oral and nasal cavities simultaneously in case of hemimaxillectomy defects $[10,11,12]$. The serratus anterior rib flap has also been described for the maxillary reconstruction, but its rib has a potential for resorption and won't accomplish the mentioned tasks [13].

As the most used donor site for vascular bone grafts, the fibula provides a surgeon with bone and soft tissue to close almost any defect in the facial region. The flap offers a long bone that can be osteotomized in many places and shaped in a desired manner. The more complex shape that has to be achieved, the longer fibula bone is needed and the shorter pedicle will remain leaving the superficial temporalis artery and vein as the only option as the recipient vessels. Mismatch of their diameters inevitably increases the risk of the flap failure. In addition, the volume of these big flaps will need a high volumetric flow rate in the recipient vessel, which lacks a superficial temporalis artery $[14,15]$.

In this article, we attempted to demonstrate the advantages of the iliac crest bone flap for hemimaxillectomy reconstruction compared to other options. With the Virtual Surgical Planning and Surgical Cutting Guides, 


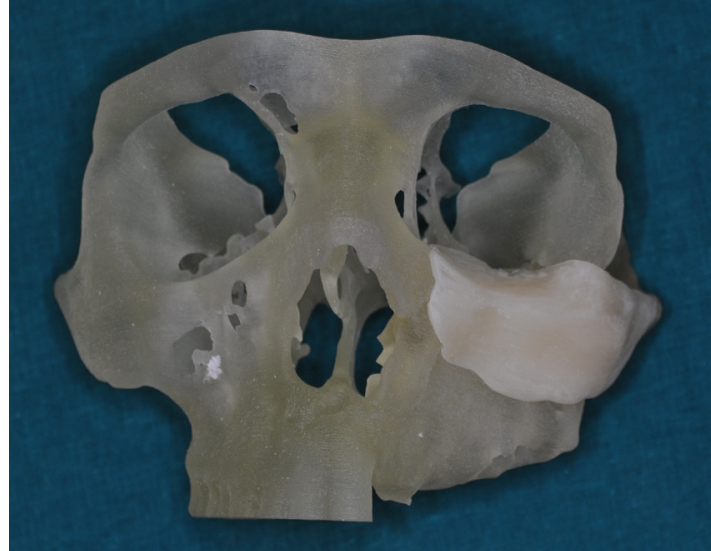

Figure 11. Stereolithographic model of the skull with the ultrahigh molecular weight polyethylene implant on it.

the iliac bone flap can be shaped to support an eye globe and be a basis for dental implants at the same time. Compared to this flap, the fibular free flap should be osteotomized in three fragments to perform the same functions. That means prolonged operation time and a risk of complications. In addition, the straight fibula cannot replicate curved orbital floor. A fragment of the fibula free flap placed at the defect of the lower orbital ridge provides insufficient support to the eye globe, prompting the utilization of the titanium mesh.

The iliac bone with the muscle cuff rarely provides sufficient tissue to close the remaining palate defect. The deep fascia and/or the inner oblique muscle can be included into the iliac crest bone flap to serve as an additional material to close intraoral defects. The skin island is too thick for this purpose. The fascia is covered with mucosa in about three weeks. In our opinion, the only possible drawback of this flap is its inability to restore the contour of the maxilla and the neighboring cheekbone precisely. This drawback, as well as the position of the eye globe, can be easily corrected by means of customized contour implants.

\section{Conclusion}

We present a revised application of the composite iliac crest bone free flap for hemimaxillectomy defects. This flap solely can give a support to the eye globe and the dental implants without osteotomies and titanium meshes. The upper ridge of the flap can be shaped to replicate the curvature of the orbital floor. The inner oblique muscle and the deep fascia can serve as an additional material for separation of the oral cavity from the nasal airway. Utilization of the Customized Contour Implants gives a surgeon a refining instrument for aesthetic correction of facial projection.

\section{Article Information}

* Correspondence: Sergey V. Tereshchuk, MD

Center for Maxillofacial Surgery and Dentistry, Burdenko Main Military Clinical Hospital, 105229, Moscow, Gospitalnaya Ploschad 3a, Russia. Email: tereschuksv@gmail.com.

Received: Apr. 23, 2017; Accepted:Jun. 25, 2018; Published: Dec. 31, 2018

DOI: 10.24983/scitemed.imj.2018.00092

Copyright (c) 2018 The Author (s). This is an open-access article distributed under the terms of the Creative Commons Attribution 4.0 International License (CC-BY).
Funding: None

Conflict of Interest: None

\section{Acknowledgments}

The authors thank the team of the Institute on Laser and Information Technologies of the Russian Academy of Sciences (ILIT RAS) and Scientific Research Center for Technological Lasers of the Russian Academy of Sciences for their invaluable assistance, including creating all computer images and medical models for this article.

\section{Informed Consent}

Informed consents were obtained from the patients for publication of the case reports and any accompanying images.

\section{Keywords}

Hemimaxillectomy; iliac crest bone flap; maxillary defect; maxillary reconstruction; virtual surgical planning.

\section{References}

1. Shrime MG, Gilbert RW. Reconstruction of the midface and maxilla. Facial Plast Surg Clin North Am 2009;17(2):211-223.

2. Brown JS, Rogers SN, McNally DN, Boyle M. A modified ctassifcation for the maxiliectomy defect. Head Neck 2000;22(1):17-26.

3. Coskunfirat OK, Wei FC, Huang WC, Cheng MH, Yang WG, Chang YM. Microvascular free tissue transfer for treatment of osteoradionecrosis of the maxilla. Plast Reconstr Surg 2005;115(1):54-60.

4. Dalgorf $D$, Higgins K. Reconstruction of the midface and maxilla. Curr Opin Otolaryngol Head Neck Surg 2008;16(4):303-311.

5. Singh M, Bhushan A, Kumar N, Chand S. Obturator prosthesis for hemimaxillectomy patients. Nat/J Maxillofac Surg 2013;4(1):117-120.

6. Tsuchiya AM, Ueno T, Taniguchi H, Ohyama T. Mobility of the obturator prosthesis in hemimaxillectomy edentulous patients. J Med Dent Sci 1998;45(1):19-27.

7. Genden EM, Wallace DI, Okay D, Urken ML. Reconstruction of the hard palate using the radial forearm free flap: indications and outcomes. Head Neck 2004;26(9):808-814.

8. Futran ND, MendezE. Developments in reconstruction of midface and maxilla. Lancet Oncol 2006;7(3):249-258.

9. Baliarsing AS, Kumar VV, Malik NA, B DK. Reconstruction of maxillectomy defects using deep circumflex iliac artery-based composite free flap. Oral Surg Oral Med Oral Pathol Oral Radiol and Endod 2010;109(3):e8-e13.

10. Ilankovan V, Ramchandani P, Walji S, Anand R. Reconstruction of maxillary defects with serratus anterior muscle and angle of the scapula. Br J Oral Maxillofac Surg 2011;49(1):53-57.

11. Kalavrezos N, Hardee PS, Hutchison IL. Reconstruction of throughand-through osteocutaneous defects of the mouth and face with subscapular system flaps. Ann R Coll Surg Eng/ 2005;87(1):45-52.

12. Valentini V, Gennaro P, Torroni A, et al. Scapula free flap for complex maxillofacial reconstruction. J Craniofac Surg 2009;20(4):1125-1131.

13. Kim PD, Blackwell KE. Latissimus-serratus-rib free flap for oromandibular and maxillary reconstruction. Arch Otolaryngol Head Neck Surg 2007;133(8):791-795.

14. Peng X, Mao C, Yu GY, Guo CB, Huang MX, Zhang Y. Maxillary reconstruction with the free fibula flap. Plast Reconstr Surg 2005; 115(6):1562-1569.

15. Zhang WB, Wang Y, Liu XJ. Reconstruction of maxillary defects with free fibula flap assisted by computer techniques. J Craniomaxillofac Surg 2015;43(5):630-636. 